\title{
Tricuspid Valve Annulus
}

National Cancer Institute

\section{Source}

National Cancer Institute. Tricuspid Valve Annulus. NCI Thesaurus. Code C130047.

A fibrous membrane that attaches to, and provides support for, the tricuspid valve leaflets. 